\title{
A Method To Measure EMI Due To Electric Field Coupling On PCB
}

\author{
Bryan M.H. Pong Angus C.M. Lee \\ Department of Electrical \& Electronic Engineering, Hong Kong University \\ Pokfulam Road, HONG KONG \\ Fax : +852-25598738, Phone : +852-28597099 \\ E-mail : mhp@hkueee.hku.hk
}

\begin{abstract}
A new method to measure electric field coupling between PCB copper traces is presented. This method enables measurement of noise from waveforms obtainable from breadboard prototypes, which is available in an early stage in the design process. The measurement result can then be analyzed and provide information for further PCB design. This method is verified by experiments and applied to an off-line flyback converter.
\end{abstract}

\section{I . INTRODUCTION}

Electromagnetic Interference (EMI) generated by switching power supplies have received a lot of attention in the industry. More and more countries have established regulations against products which generate EMI. The switching nature of switch mode power supply makes it an inevitable source of EMI. Engineers and researchers are striving to reduce EMI by various methods. From experience it is well known that circuit layout design on a printed circuit board (PCB) plays a very important part in the reduction of EMI. A good circuit layout can very often solve EMI problems without any changes in circuit topology or components in the circuit. However, there is very little information available on the EMI performance of a switching power supply circuit until the circuit is fully laid out on a PCB and tested. Even when the circuit is breadboarded only the functional performance of the power supply can be tested, very little EMI performance information are available because the circuit layout of the breadboard prototype will be very different from the final layout. This makes overall EMI measurement in the breadboard stage meaningless. Ho:vever, it will be extremely useful for the engineer who lay the circuit on a PCB have as much information as possible on circuit parts with high emission so that these parts can be dealt with carefully. Computer modeling of the whole circuit including details of all components and $\mathrm{PCB}$ layouts is an overwhelming task even for state of the art technology. Even though one can manage the modeling and simulations, the results will definitely be too complex to understand and hard to be translated to useful information. Very often one has to resort to actual measurements.

Every EMI problem comprises three elements, namely a source, a receiver and a mechanism which couples interference from the source to the receiver as in Fig. 1

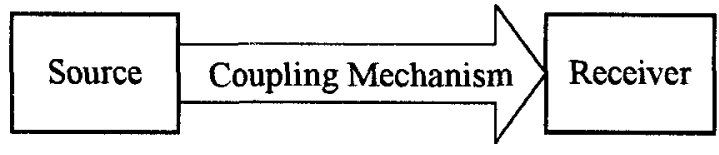

Fig.1. Basic Elements in EMI Problems

Coupling mechanism can be categorized into conducted coupling and non-conducted coupling. Conducted coupling refers to interference along a conductor which links up the source and the receiver. Non-conducted coupling comprises of electric field coupling, magnetic field coupling and a combination of both. In switching power supplies, electric field coupling has considerable contribution to overall EMI especially those circuits which involve high voltage transients. This paper describes a simple and low cost method to measure electric field coupling at circuit nodes. This method can be applied to breadboard prototypes and potential sources of electric field interferences can be identified. Measurement results will provide electric coupling information at various circuit nodes. PCB designer can make use of these information in the layout design.

\section{II . ELECTRIC FIELD COUPLING ON PCB}

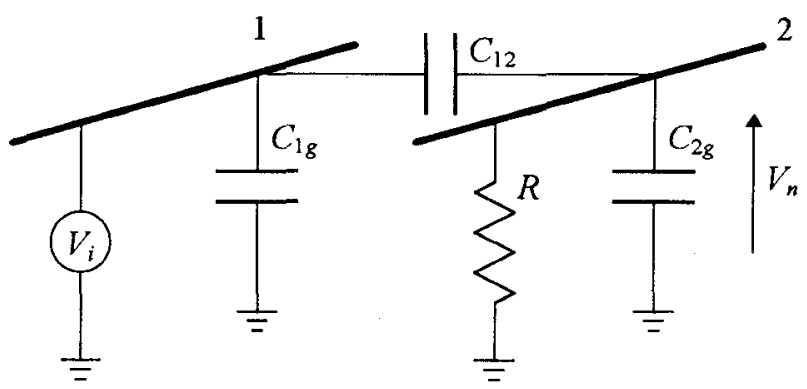

Fig.2. Electric Field Coupling Model

A simple representation of electric field coupling [1] between PCB traces is shown in Fig.2. Noise source $V_{i}$ is connected to conductor 1 and the interference noise couples to conductor 2 through capacitor $C_{12}$.

The noise voltage picked up by conductor 2 is represented by

$$
V_{n}=\frac{j \omega\left[\frac{C_{12}}{\left(C_{12}+C_{2 g}\right)}\right]}{j \omega+\frac{1}{R\left(C_{12}+C_{2 g}\right)}} V_{i}
$$


In most practical cases,

$$
R \ll \frac{1}{j \omega\left(C_{12}+C_{2 g}\right)}
$$

and (1) can be simplified to

$$
V_{n}=j \omega R C_{12} V_{1}
$$

\section{III . APPARATUS}

The principle of the measurement method is to pick up the noise source from nodes of the circuit under test, and connect it to a fixed structure which simulates electric field coupling on PCB. The coupled noise voltage on the receiver end is analyzed by a measurement instrument.

The apparatus comprises of two copper traces which are printed on a PCB. One of the traces is to be connected to the circuit under test. Another trace is to be connected to measurement instrument which is typically a spectrum analyzer. A schematic diagram of the apparatus is shown in Fig.3.

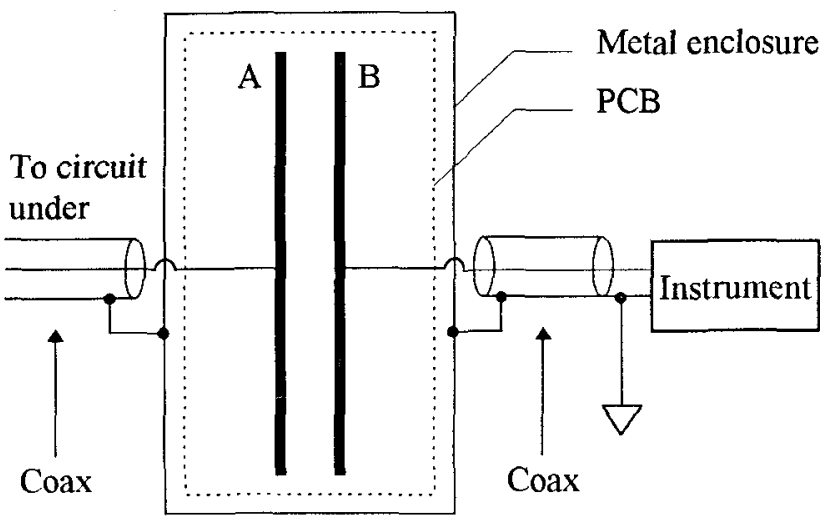

Fig.3. Schematic Diagram of Apparatus Setup

On the PCB there are copper traces A and B which are laid parallel to one another. Traces $A$ and $B$ are separated by a fixed distance. Trace $A$ is connected to the circuit under test via a coaxial cable. Trace $B$ is connected to a measurement instrument via another coaxial cable. The shield of the coaxial is grounded together with the instrument ground and the metal enclosure. There is no electrical connection between traces $A$ and $B$.

A coaxial cable from the apparatus is connected to two nodes of interest in a switching circuit by which the noise signal is brought up to the apparatus. These two nodes may be, say, the drain and the source of a MOSFET in a switching power supply circuit, or any other nodes which is likely to be noise generator. Note that the shield of the coaxial cable is connected to Ground at earth potential. So precaution must be made so that when connecting earth to the circuit under test. The signals picked up are differential mode noise between the two nodes. This can be modeled by the equivalent circuit in Fig. 2.

$V_{i}$ is the noise source which generates an electric field. The spectrum of the electric field is strongly dependent on the voltage wave shape. $C_{1 g}$ is the capacitance of the interconnection between the circuit under test and the apparatus. In this case $C_{\mathrm{lg}}$ is the capacitance the interconnection coaxial cable, plus the capacitance between trace $A$ and the metal enclosure.

$$
C_{1 g}=C_{(\operatorname{coax} \text { in })}+C_{(\mathrm{A \& M})}
$$

$C_{12}$ is the capacitance between copper trace A and copper trace $B$

$$
C_{12}=C_{(\mathrm{A} \& \mathrm{~B})}
$$

$C_{2 g}$ is the capacitance between copper trace B and the metal enclosure, plus the capacitance of the interconnection cable which connects to the measurement instrument.

$$
C_{2 g}=C_{(\mathrm{B} \& \mathrm{M})}+C_{(\text {coax out })}
$$

The spectrum analyzer to be used has an input impedance $R$ of $50 \mathrm{ohm}$, which can satisfy the condition described in (2), and hence the noise voltage in (3) is valid in this case. From (3), the noise voltage $V_{n}$ is independent of $C_{1 g}$ and $C_{2 g}$. In other words, the coaxial cable length is not a critical parameter provided that its length is much smaller than the wavelength of the harmonics to be measured.

The coupling noise will be the capacitive coupling of the noise voltage if a copper trace is laid on a PCB with a length and a distance the same as traces $A$ and $B$. Other length and distances can be obtained by correction factors.

\section{IV . EXPERIMENTAL VERIFICATION USING A SIGNAL GENERATOR}

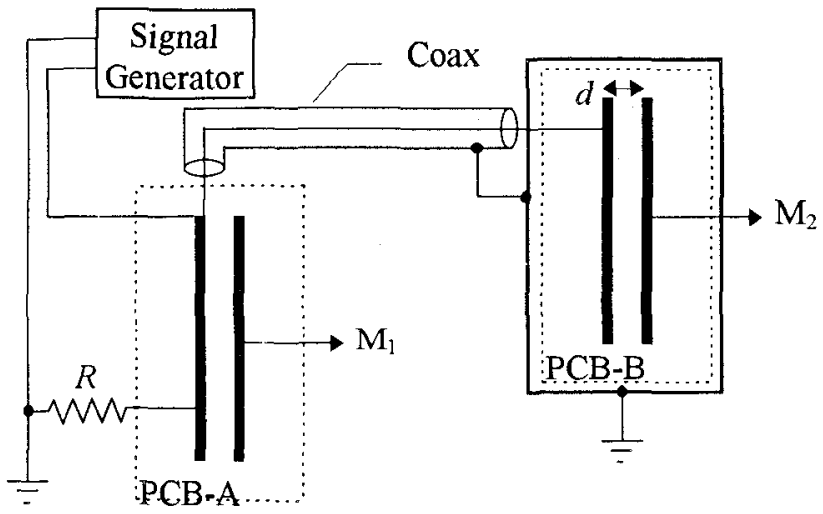

Fig.4. Experimental Verification Using a Signal Generator 
An experiment is set up to verify the principle of electric field coupling. Two identical PCB are fabricated and they are connected with a resistor as shown in Fig.4. PCB-A is connected to a signal generator through a resistor to simulate the tracks inside the $\mathrm{PCB}$ of the convertors, while PCB-B is part of the apparatus described in Fig. 3 which is housed in a metal enclosure with coaxial cables as input and output connections. The signal input to PCB-A simulates a switching waveform coupling to an adjacent copper trace inside the converter. The same signal is then transferred to the apparatus described in the previous section. Measurement are taken at $M_{1}$ and $M_{2}$ respectively and compared with each other.

The comparison of the spectrums obtained with square wave at $300 \mathrm{kHz}$ input are shown in Fig.5a and Fig.5b. It can be seen that the two spectrum closely resemble each other and this shows that the electric field coupling between internal traces can be represented through the connection of a pair of external parallel traces. Hence the measurement method in the previous section is validated.

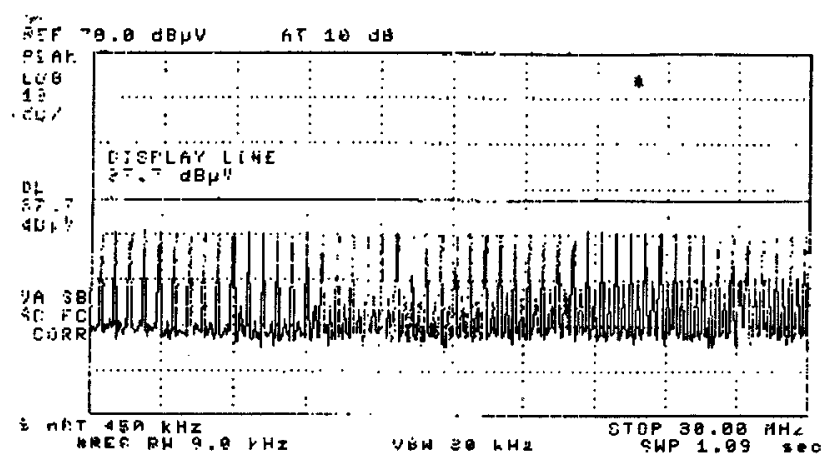

(a) Spectrum Measured at $M_{1}$

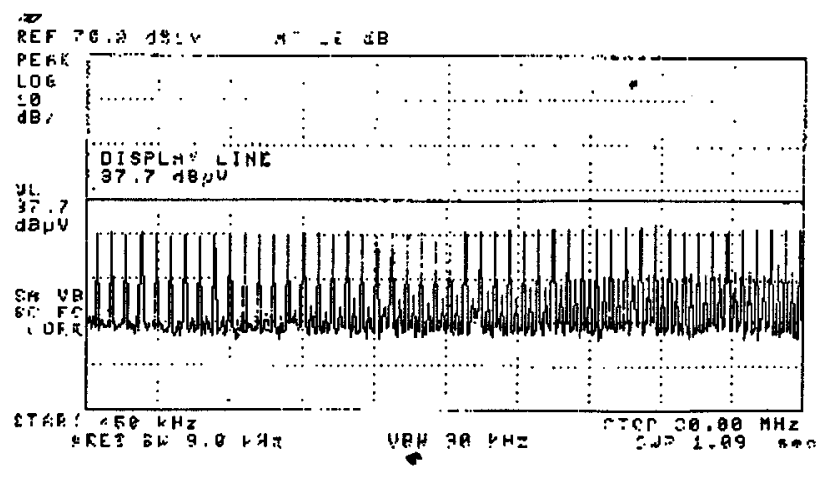

(b) Spectrum measured at $\mathrm{M}_{2}$

Fig.5. Spetrum measured with a square wave input from signal generator. trace length $l=4 \mathrm{~mm}$, trace separation $d=3 \mathrm{~mm}$, trace width $w=1 \mathrm{~mm}$ frequency $=300 \mathrm{kHz}$, amplitude $=2 \mathrm{~V}_{\mathrm{p}-\mathrm{p}}$

A more precise measurement is achieved by generating a sine wave and apply to the same circuit as shown in Fig.4. As the sine wave contains a single frequency only, an unique peak is detected in the spectrum analyzer. The peak values at $M_{1}$ and $M_{2}$ are recorded for different input frequency.
Table $I$ indicates the coupling voltages at $M_{1}$ and $M_{2}$ match closely with less than $1 \mathrm{~dB} \mu \mathrm{V}$ inside the measuring frequency range. This further confirms the validity of the measuring technique.

TABLE I

PEAK Voltage Recorded WITH SINE WAVE INPUT

\begin{tabular}{ccc}
\hline $\begin{array}{c}\text { Frequency } \\
(\mathrm{MHz})\end{array}$ & $\begin{array}{c}\text { Voltage at } \mathrm{M}_{1} \\
(\mathrm{~dB} \mu \mathrm{V})\end{array}$ & $\begin{array}{c}\text { Voltage at } \mathrm{M}_{2} \\
(\mathrm{~dB} \mu \mathrm{V})\end{array}$ \\
\hline 1 & 32.1 & 32.8 \\
5 & 45.5 & 45.8 \\
10 & 51.7 & 52.5 \\
\hline
\end{tabular}

trace length $l=54 \mathrm{~mm}$, trace separation $d=2 \mathrm{~mm}$, trace width $w=1 \mathrm{~mm}$, signal $=$ sine wave amplitude $=1 \mathrm{~V}_{\mathrm{p}-\mathrm{p}}$

In practice, the traces may have different track length $l$ track width $w$ and track separation $d$. It affects the capacitance $C_{12}$ mainly because the characteristics follow (2) in most practical case. Walker [1] gives the equation for the variation in these parameters to the effect of $C_{12}$

$$
C_{12}=\frac{27.8 \varepsilon_{r(e f f)}}{\ln \left[\frac{\pi(d-w)}{w+t}+1\right]} \times l
$$

where $l$ is the trace length in meter, $d$ is the trace separation in meter, $w$ is the trace width in meter and $t$ is the trace thickness in meter.

For a new set of capacitance $C_{12}^{\prime}$ and coupling voltage $V_{n}^{\prime}$

$$
\frac{C_{12}^{\prime}}{C_{12}}=\frac{\ln \left[\frac{\pi(d-w)}{w+t}+1\right]}{\ln \left[\frac{\pi\left(d^{\prime}-w^{\prime}\right)}{w^{\prime}+t^{\prime}}+1\right]} \times \frac{l^{\prime}}{l}=\frac{V_{n}^{\prime}}{V_{n}}
$$

The correction factor $F$ can be expressed in $\mathrm{dB}$ as

$$
\mathrm{F}=20 \log \left[\frac{l^{\prime}}{l} \frac{\ln \left[\frac{\pi(d-w)}{w+t}+1\right]}{\ln \left[\frac{\pi\left(d^{\prime}-w^{\prime}\right)}{w^{\prime}+t^{\prime}}+1\right]}\right]
$$

and

$$
20 \log V_{n}^{\prime}-20 \log V_{n}=\mathrm{F}
$$

or

$$
V_{n}^{\prime}-V_{n}=\mathrm{F} \text { in } \mathrm{dB}
$$

The experimental setup in Fig.4 is also used to verify the validity of the scaled coupling effect of (11). A sine wave is generated from the signal generator, instead of a square wave. It is conducted with the tracks with different 
separation $d$. The reference voltage $V_{n}$ 'is measured with a pair of arbitrary traces. Then other the coupling voltage of other traces are recorded as $V_{n}$ in Table II.

According to (11), the correction factor $F$ is equal to the difference between the $V_{n}^{\prime}$ and $V_{n}$. Close matching of measured and calculated results in Table II shows that the correction factor $F$ can perform the trace transformation in a high degree of accuracy, given that the trace separation $d$ is small comparing with trace length $l$.

TABLE II

EXPERIMENTAL VERIFICATION OF CORRECTION FACTOR F

\begin{tabular}{|c|c|c|c|c|}
\hline & & & Measured & Calculated \\
\hline $\begin{array}{l}\text { Trace sepa- } \\
\text { ration } d \\
(\mathrm{~mm})\end{array}$ & $\begin{array}{c}\text { Voltage at } \\
\mathrm{M}_{2} V_{n}^{\prime} \\
(\mathrm{dB} \mu \mathrm{V})\end{array}$ & $\begin{array}{c}\text { Reference } \\
\text { voltage } V_{n} \\
(\mathrm{~dB} \mu \mathrm{V})\end{array}$ & $\begin{array}{l}V_{n}^{\prime}-V_{n} \\
(\mathrm{~dB} \mu \mathrm{V})\end{array}$ & $\begin{array}{c}\text { Calculated } \\
\text { F } \\
(\mathrm{dB} \mu \mathrm{V}) \\
\end{array}$ \\
\hline 5 & 37.5 & 29.7 & 7.8 & 7.1 \\
\hline 10 & 31.3 & 29.7 & 1.6 & 1.6 \\
\hline 20 & 27.1 & 29.7 & -1.4 & -1.3 \\
\hline
\end{tabular}

trace thickness $t=0.1 \mathrm{~mm}$, frequency $=1 \mathrm{MHz}$, amplitude : $1 \mathrm{~V}_{\mathrm{p}-\mathrm{p}}$

$V_{n}$ :

trace length $l=54 \mathrm{~mm}$, trace width $w=2 \mathrm{~mm}$, trace separation $d=1 \mathrm{~mm}$

$V_{n}^{\prime}$ :

trace length $l^{\prime}=100 \mathrm{~mm}$ Trace width $w^{\prime}=3 \mathrm{~mm}$

\section{V . EXPERIMENT WITH AN LISN AND A FLYBACK CONVERTER}

This experiment demonstrates the application of this measuring technique on a flyback converter. A typical flyback converter comprises a bridge rectifier, a flyback transformer, a MOSFET and a series of filter capacitors as shown in Fig.6. The significant node pairs are labeled from A to F. A coaxial cable is connected two nodes, for example $\mathrm{A}+$ and $\mathrm{A}-$, so that the noise voltage across $\mathrm{A}+$ and $A$ - is brought up to one side of the parallel traces. The other side of the parallel traces is connected to a spectrum analyzer for measurement. Fig. 7 describes the experimental setup. The shielding of the coaxial cables connects to each other at the metal enclosure such that external noise is inhabited in this environment. It is noted that the converter circuit and the measuring circuit should be isolated. The differential mode noise waveform from nodes $\mathrm{A}$ to $\mathrm{F}$ observed at the spectrum analyzer is recorded in Figs.8(a) to Fig.8(f). The spectrum across nodes $\mathrm{A}+$ and $\mathrm{A}-$ shows a very low noise level as it is the input side of the converter, in which no switching noise is presented. Also the capacitor across $A+$ and $A-$ filters out the surge in the input. Same situation is found at nodes B+ and B- as well since it does not produce high frequency noise through the bridge rectifier. Nodes $\mathrm{C}+$ and $\mathrm{C}$ - are electrically equivalent to nodes $\mathrm{B}+$ and $\mathrm{B}-$ so that a low noise level is expected. The most significant noise comes from the switching of the MOSFET. Fig.8(d) shows the noise spectrum of node D+ and D- which is measured across the drain and source of the switch MOSFET; a very high noise level is found. The switch noise is coupled through the transformer to the sec- ondary side of the converter. The noise voltage is recorded across nodes $\mathrm{E}+$ and $\mathrm{E}$ - as shown in Fig.8(e). It shows a

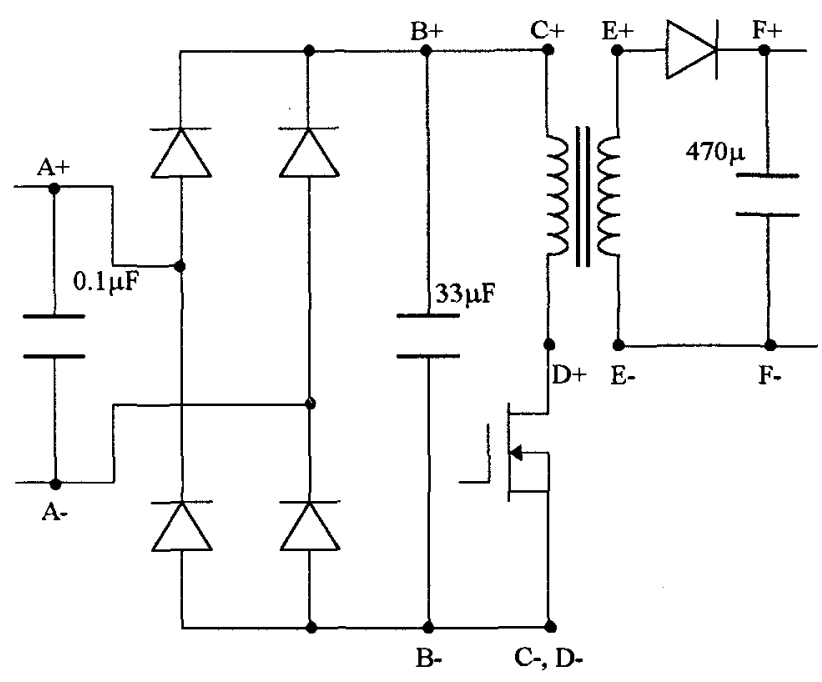

Fig.6. Schematic diagram of a typical Flyback Converter

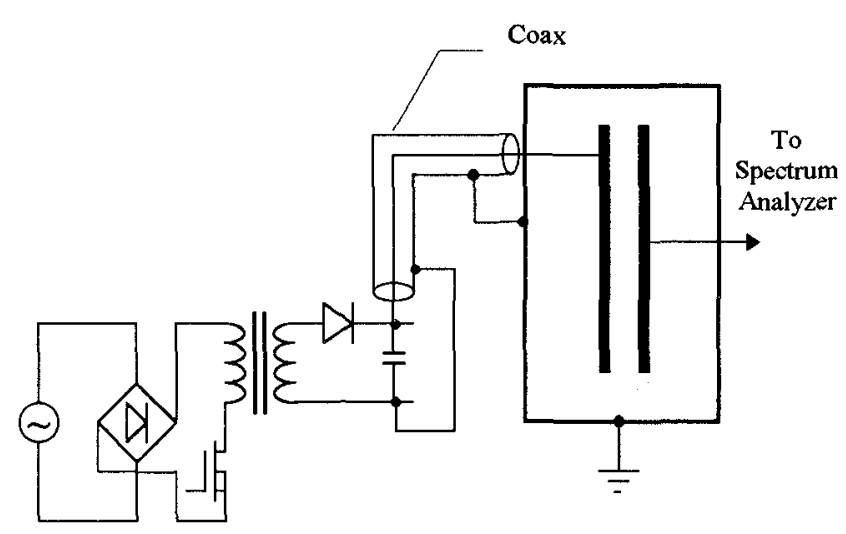

Fig.7. Experimental Setup Using a Flyback Converter

medium noise level throughout the whole measuring frequency. The noise is suppressed by the capacitor across nodes $\mathrm{F}+$ and $\mathrm{F}$ - to produce a low noise spectrum.

The experiment verifies the critical noise source in the flyback converter circuit. Both nodes $D$ and $E$ are strong sources of noise. Although the noise level at node $\mathrm{E}$ is not as high as that at node $\mathrm{D}$ in general, a wide frequency range of noise is observed. PCB designer should pay attention to these important nodes.

The converter is then tested for overall conducted noise using the standard noise measurement method through a line impedance stabilization network (LISN) and the observed spectrum is shown in Fig.9. Comparing the spectrum obtained through the LISN, the spectrum obtained at nodes D+ and D- represents a very similar profile. This result shows that the conducted noise is highly affected by the electric field coupling from the traces connected to these switching points.

The previous section verifies the relationship between the electric field coupling and traces dimension. The result can be applied together with the spectrums obtained to 
provide information on optimum trace separation in the PCB layout, such that electric field coupling is suppressed in the circuit.

The peak voltage between the PCB traces may approach $500 \mathrm{~V}$ in the switching of power electronic devices. The high surge can cause flash-over between the traces and damage the spectrum analyzer or other devices. Therefore one should take a note on the separation between the two PCB traces.

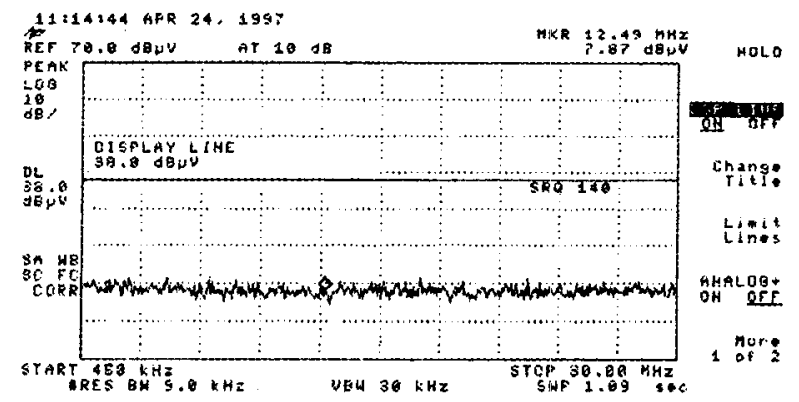

(a) Spectrum measured at point $\mathrm{A}$

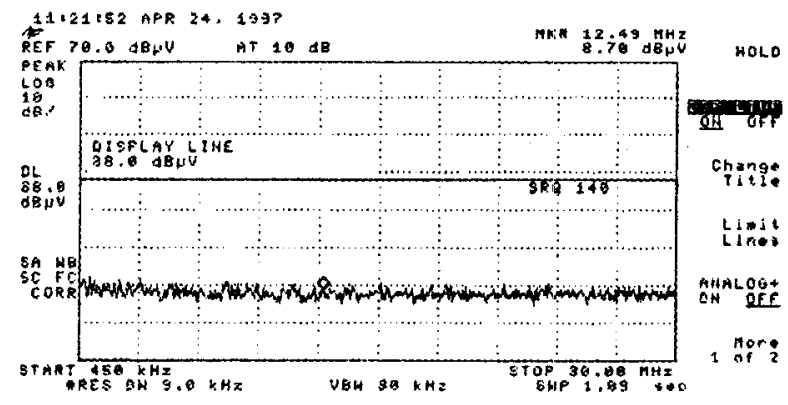

(b) Spectrum measured at point B

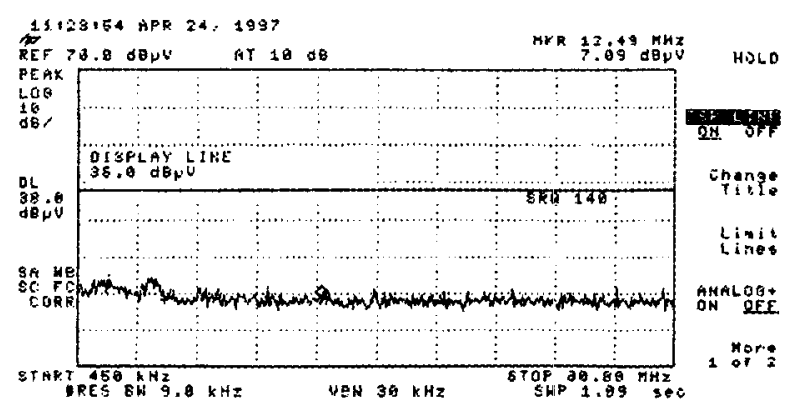

(c) Spectrum measured at point $\mathrm{C}$

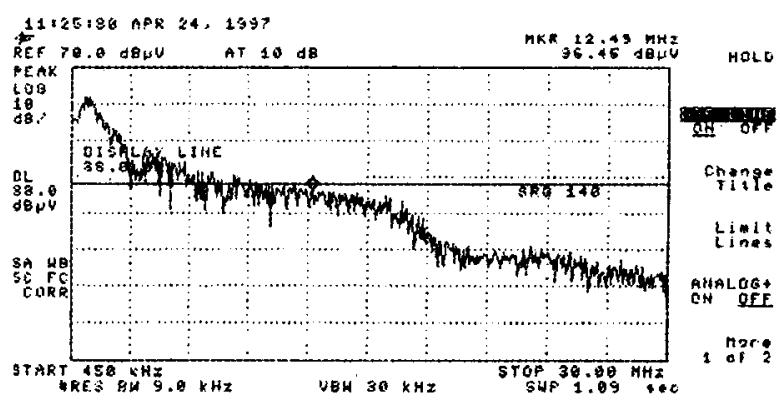

(d) Spectrum measured at point D

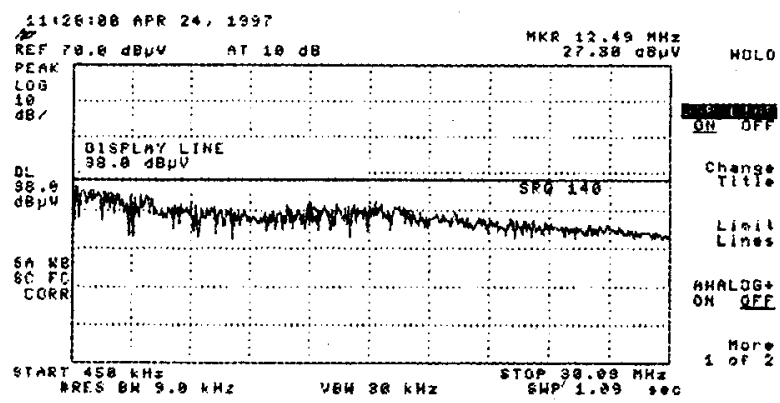

(e) Spectrum measured at point $\mathrm{E}$

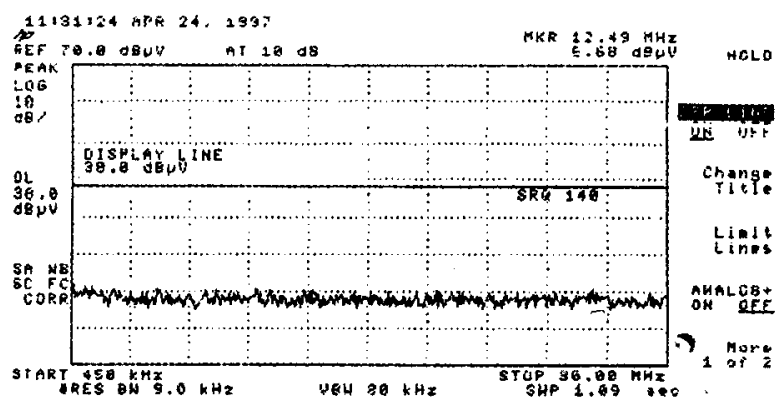

(f) Spectrum measured at point $F$

Fig.8. EMC Measurement of a Flyback Converter

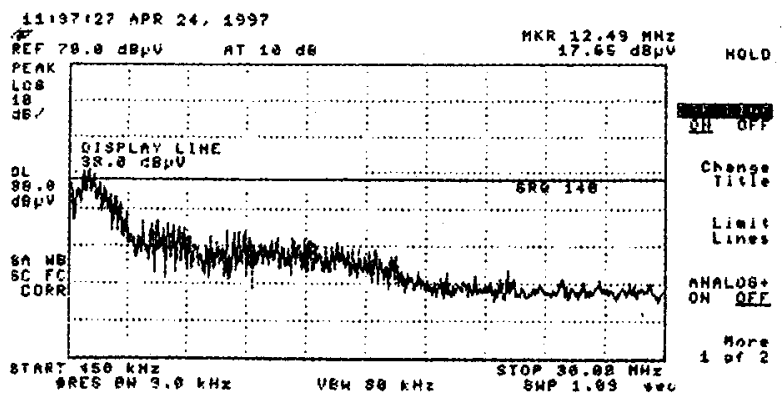

Fig.9. Spectrum Measured at Line Input Thraugh a LISN 


\section{VI . CONCLUSION}

This paper describes a new method to estimate EMI due to electric field coupling between PCB traces in which its effect is significant in the switching nature of the model power electronic system. A simple set of apparatus is required and the test can be achieved easily. Experimental results prove the accuracy of this method.

Moreover, it provides a means for engineers to estimate the electric field coupling between copper traces from spectrums which are obtainable from prototypes. This provides useful information to PCB designer on the required dimensions and separation of trace layout and avoids EMI problems in an early stage in the design process, such that the PCB design can still be changed in a convenience way.

\section{REFERENCES}

[1] C. S. Walker, Capacitance, Inductance and Crosstalk Analysis. Artech House, 1990.

[2] H. W. Ott, Noise Reduction Techniques in Electronic Systems. $2^{\text {nd }}$ Edition, Wiley, New York, 1988.

[3] M. Mardiguian, Controlling Radiated Emissions by Design. Van Nostrand Reinhold.

[4] L. Tihanyi, Electromagnetic Compatibility in Power Electronics. IEEE Press, 1995.

[5] L. B. Gravelle and P. F. Wilson, "EMI/EMI in Printed Circuit Boards - A Literature Review," IEEE Transactions on Electromagnetic Compatibility, vol. 34, no. 2, May 1992.

[6] J. C. Fluke, Sr., Controlling Conducted Emission by Design. Van Nostrand Reinhold.

[7] C. R. Paul, Introduction to Electromagnetic Compatibility. $1^{\text {st }}$ Edition, Wiley, New York, 1992.

[8] T. N. Bahr and E. J. MacMahon, Electrostatic Discharge Control. Hayden, New York, 1983.

[9] A. N. Faught, "An Introduction to Shield Joint Evaluation using EMU Gasket Transfer Impedance Data," in Proceedings of the IEEE Symposium, pp. 38-44, 1982.

[10] J. Goedbloed, Electromagnetic Compatibility. Prentice Hall, New York, 1990.

[11] R. J. Mohr, "Evaluation Technique for EMI Seams," in Proceedings of the IEEE Symposiumon Electromagnetic Compatibility, pp. 8184, 1987.

[12] B. Keiser, Principles of Electromagnetic Compatibility, $3^{\text {rd }}$ Edition, Aertech House, Norwood, MA, 1987.

[13] E. D. Knowles and L. W. Olson, "Cable Shielding Effectiveness Testing." IEEE Transaction on Electromagnetic Compatibility, EMC-16, February, 1974

[14] C. R. Paul and K. B. Hardin, "Diagnosis and Reduction of Conducted Emissions," in Proceeding of the IEEE Symposium on Electromagnetic Compatibility, pp. 19-23, 1988.

[15] C. R. Paul and W. W. Everett III, "Printed Circuit Board Crosstalk," in Proceedings of the IEEE Symposium on Electromagnetic Compatibility, pp. 452-459, 1985.

[16] J. L. N. Violette, D. R. J. White and M. F. Violette, Electromagnetic Compatibility Handbook, Van Nostrand Reinhold, New York, 1987. 